\title{
Configurable 2-D Linear Feedback Shift Registers for VLSI Built-in Self-test Designs
}

\author{
CHIEN-IN HENRY CHEN* and YINGJIE ZHOU \\ Department of Electrical Engineering, Wright State University, Dayton, $\mathrm{OH} 45435$
}

(Received 20 May 1999; In final form 13 October 1999)

\begin{abstract}
Recently a multiple-sequence test generator was presented based on two-dimensional linear feedback shift registers (2-D LFSR). This generator can generate a set of precomputed test vectors obtained by an ATPG tool for detecting random-pattern-resistant faults and particular hard-to-detect faults. In addition, it can generate better random patterns than a conventional LFSR. In this paper we describe an optimized BIST scheme which has a configurable 2-D LFSR structure. Starting from a set of stuck-at faults and a corresponding set of test vectors detecting these faults, the corresponding test pattern generator is determined automatically. A synthesis procedure of designing this test generator is presented. Experimental results show that the hardware overhead is considerably reduced compared with 2-D LFSR generators.
\end{abstract}

Keywords: Linear feedback shift registers (LFSR); built-in self-test (BIST); pseudo-random testing; deterministic testing; fault detection and fault coverage

\section{INTRODUCTION}

Ever-increasing VLSI circuit density has made today's chip testing more complicated and costly. Built-in self-test (BIST) has been proven to be an effective approach to achieve sufficiently high, if not complete, fault coverage without any external automatic test equipment on site. It has revolutionized the way the integrated circuit chips can be tested by (1) reducing the cost of manufacturing testing by shortening the test application time, (2) minimizing the amount of test data stored, and (3) lowing the cost of test equipment. The implementation of BIST can result in a reduction of the product development cycle and cost, and the cost of system maintenance can be significantly reduced by testing chips, boards, and the entire system virtually using BIST.

Typical BIST architecture consists of a test pattern generator and a test response analyzer. The efficiency of BIST implementation can be characterized by test length and hardware overhead for a given fault coverage requirement which is defined as the ratio of the number of faults that can be detected to the total number of faults in the assumed fault domain. It is expected that BIST

\footnotetext{
*Corresponding author.
} 
insertion to integrated circuit chips will result in high fault coverage, small volume of test data, at-system test speed, and compatibility with the design for testability techniques. High fault coverage can be achieved only if all faults of interest are detected and their effects are retained in the test response analyzer.

There have been various BIST techniques based on pseudo-random testing $[1,9]$, pseudo-exhaustive testing $[2,10,11]$, weighted random testing $[3,12,13]$, and reseeding of LFSR [5-7]. These approaches offer different trade-off among test data volume, test application time, hardware overhead and fault coverage. One of the most popular approaches is the pseudo-random BIST, which employs an LFSR to generate a large number of test patterns with small hardware overhead. The major limitations with this method are the long test time and low fault coverage. Several approaches have been proposed to overcome these disadvantages, most of which embed a set of precomputed test vectors, called deterministic patterns, to reach high fault coverage within a shorter time. Such deterministic patterns can be obtained by an automatic test pattern generation (ATPG) and implemented with a ROM to store the patterns, or the traditional LFSRs with pre-designed seeds to generate the patterns.

Recently, another method was presented to realize both pseudo-random pattern generation and deterministic pattern embedding using a 2-D LFSR [8]. With the two-dimensional feedback algorithm, it can achieve higher fault coverage than the conventional LFSR due to improved randomness. However, the hardware overhead is much higher than conventional LFSR for some practical circuits. In this paper, our focus is to reduce the hardware overhead by proposing a configurable 2-D LSFR based BIST scheme.

\section{2-D LINEAR FEEDBACK SHIFTER REGISTERS}

A conventional LFSR can be used to generate a large number of pseudo-random test patterns with very small area overhead that is composed of exclusive-or gates (XORs) and flip-flops (FFs). The general structure is shown in Figure 1, which can be expressed with the polynomial in Eq. (1).

$$
P(V)=1+C_{1} V+C_{2} V^{2}+\cdots+C_{M-1} V^{M-1}+V^{M}
$$

The coefficients $C i(i=1 \sim M-1)$ are zero or one. An LFSR goes through a cyclic sequence of states and the outputs produced are also periodic. The maximum length of this period is $2^{M}-1$, where $M$ is the number of stages. So the patterns generated by an LFSR are pseudo-random and the random properties depend on its initial state and its generating function $P(V)$. Once the generating function and the initial state are fixed, the patterns produced are also determined and in one period there is no recurrent patterns. It cannot produce a sequence of deterministic ordered patterns. The fault coverage is usually low because the randompattern-resistant faults exist in some logic structures that can be detected only by deterministic ordered patterns. A number of the LFSR based techniques [5-7] have been developed to generate deterministic ordered test patterns to detect the random-pattern-resistant faults, but the encoding and decoding procedures and the implementation of control logic are relatively complex. A 2-D LFSR structure was presented in [8], which can first generate a deterministic sequence used to detect random-pattern-resistant faults, followed by random patterns used to detect a large percentage of easily testable faults. Figure 2 shows the general representation of a 2-D LFSR based on $N$ primitive polynomials:

$$
V_{i}=\sum_{j=1}^{N} \sum_{k=1}^{M} a_{i j k} V_{j} D^{k}+C_{i}, \quad i=1 \sim N
$$

This circuit consists of $N$ shift registers, each of which has $M$ stages. $V_{i}(i=1 \sim N)$ represents an $N$ bit vector, and $V_{i} D^{k}(k=1 \sim M)$ represents the $k$ th delay of a vector $V_{i} . \quad V_{i}$ is generated by the feedback network given by Eq. (2). If $a_{i j k}=1$, the 


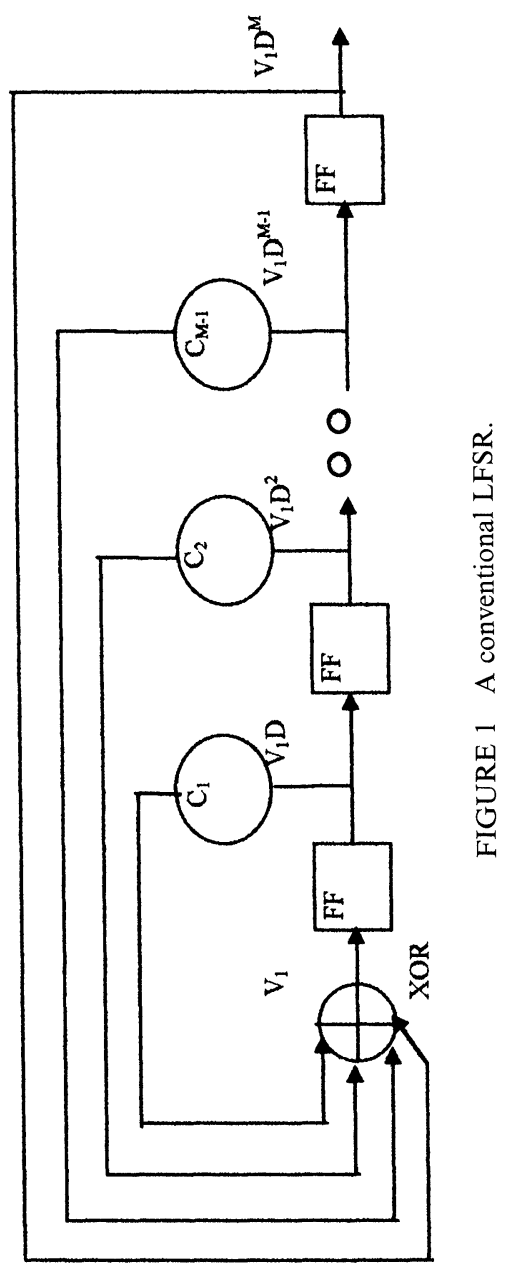




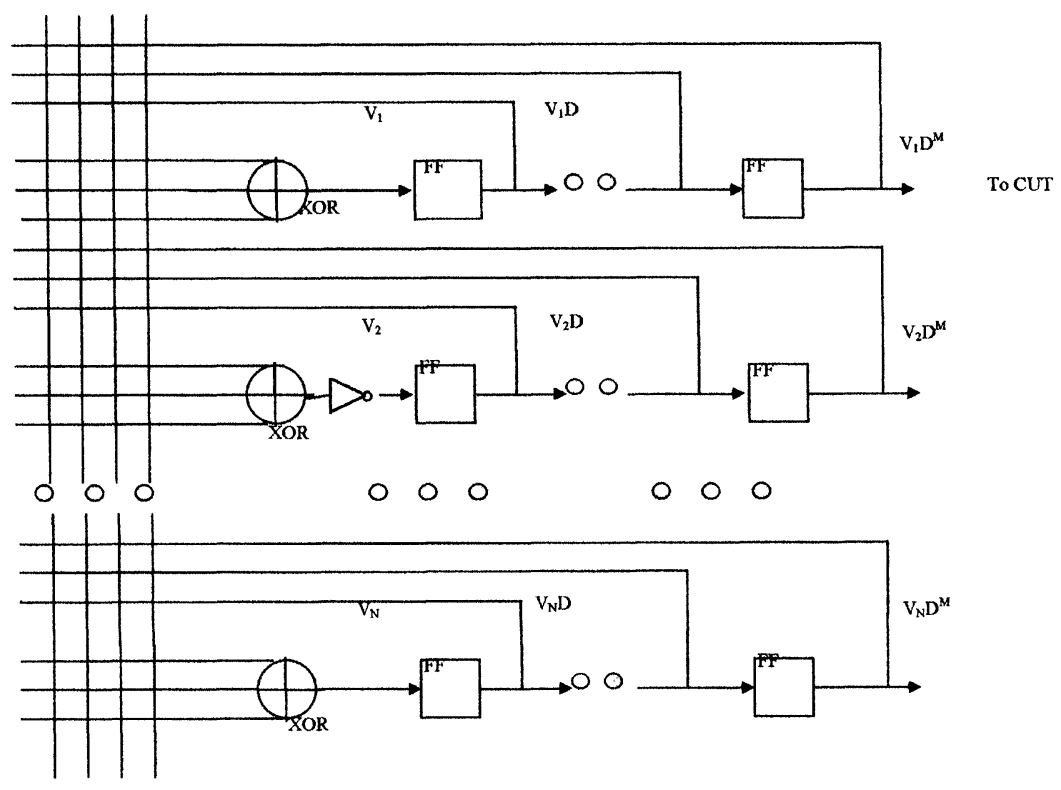

FIGURE 2 Two dimensional LFSR structure.

signal $V_{j} D^{k}$ is connected to the XOR gate to generate $V_{i}$. Otherwise, there is no connection. If $C_{i}=0$, the output of the XOR gate is connected to the shift register directly. If $C_{i}=1$, then an inverter is added to the input of the shift register for generating a complementary feedback signal. Given $L$ vectors with a length of $N$-bit,

$$
\begin{gathered}
V_{1}: b_{11}, b_{12}, b_{13}, \ldots, b_{1 L} \\
V_{2}: b_{21}, b_{22}, b_{23}, \ldots, b_{2 L} \\
\ldots \\
V_{N}: b_{N 1}, b_{N 2}, b_{N 3}, \ldots, b_{N L} .
\end{gathered}
$$

To store these vectors in a 2-D LFSR with minimum stage $M$, we construct Eq. (3) and obtain the solutions including determining the minimum value of $M$ by using the branch and bound method.

$$
V_{i}(n)=\sum_{j=1}^{N} \sum_{k=1}^{M} a_{i j k} V_{j}(n-k)+C_{i}
$$

where $V_{i}(n)=b_{i, n}, \quad V_{j}(n-k)=V_{j}(n) D^{k}=b_{j,(n-k)}$, $i=1 \sim N$ and $n=1 \sim L$. When solving the first vector $V_{i}(1), V_{j}(1-k)$ represents the initial states of the 2-D LFSR. A procedure to solve Eq. (3) is described below:

\{ $\mathrm{M}=1 ; \quad / \star \mathrm{M}$ is the index count of time frame expansions $\star 1$

Set initial states of 2-D LFSR;

While (aijk's for any signal $V i$ are not found) \{ if ( $a_{i j k}$ 's for each signal $V_{i}$ are found) return;

for (each unsolved $V_{i}$ signal) \{

Build_Equations; / $*$ Formulate Eq. (3) and set $C_{i}=0 * /$

Assign a set of $a_{i j k}$ values to Eq. (3);

if ( $a_{i j k}$ values are valid) mark signal $V_{i}$ SOLVED; else \{

use branch \& bound method to obtain a new set of $a_{i j k}$ values;

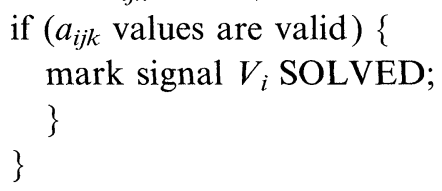


if ( $V_{i}$ is not SOLVED ) \{

Build_Inverted_Equations; /* For-

mulate Eq. (3) and set $C_{i}=1 * 1$

Assign a set of $a_{i j k}$ values to Eq. (3);

if ( $a_{i j k}$ values are valid)

mark signal $V_{i}$ SOLVED;

else \{

use branch \& bound method to

obtain a new set of $a_{i j k}$ values;

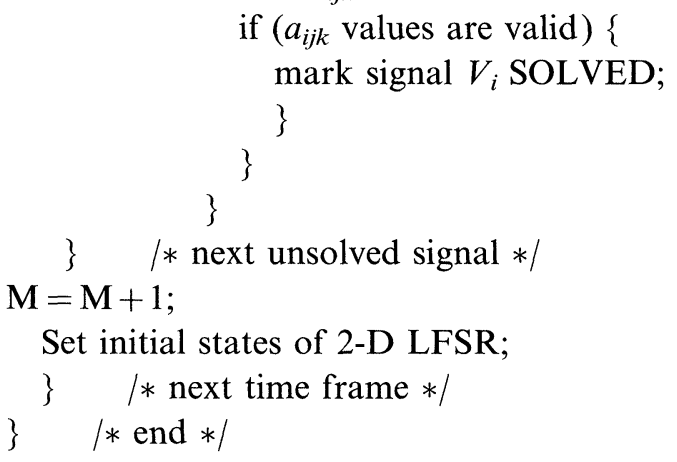

Besides embedding the deterministic sequence, the 2-D LFSR can generate better pseudo-random patterns than a conventional LFSR according to the experimental results in [8]. The longer the deterministic sequence is, the more stages of a 2-D LFSR are required, which means more hardware required. For some practical circuits, a large overhead is unacceptable. In the next section, a feasible configuration scheme based on a 2-D LFSR to reduce the overhead is proposed.

\section{CONFIGURABLE 2-D LFSR BASED TEST PATTERN GENERATOR}

A configurable 2-D LFSR based test pattern generator is proposed to generate an embedded deterministic sequence of test patterns followed by pseudo-random patterns. The generator mainly consists of four types of function blocks - the flipflop array (FFA), the configuration network (CN), the multiplexers (MUXs), and the control unit (CU) as shown in Figure 3. The FFA is an $N \times M$ flip-flop array, where $N$ is the number of inputs of a circuit under test (CUT) and $M$ is the number of stages of the 2-D LFSR. In order to reduce the hardware, $M$ is usually a small number. If Eq. (3) cannot be solved with a small value of $M$, the embedded test sequence is then partitioned into subsequences for obtaining solutions for a configurable scheme. Each $\mathrm{CN}$ consists of XOR gates and an inverter if necessary. The MUX block selects one of the $p$ configuration networks and feeds the feedback signal to the $N$-input FFA, where $p$ is the number of configurations. The MUX block is controlled by $\left[\log _{2} p\right]$ signals as shown in Figure 3. A relatively simple CU generates these signals for the MUX and a reset signal for the FFA. When resetting the generator, the initial states of FFA are set to $\langle 1010 \cdots\rangle$ in each column of the FFA.

The synthesis procedure for a configurable 2-D LFSR is shown in Figure 4 with two main tasks: (1) find a set of deterministic ordered patterns and (2) embed these patterns in the configurable 2-D LFSR. The HITEC [4] is used as an ATPG tool to generate a set of test patterns for detecting random-pattern-resistant faults. For some circuits, however, it is hard to find test patterns to detect random-pattern-resistant faults only. Therefore, all faults including random-pattern-detectable and random-pattern-resistant faults are considered in ATPG. With the test patterns generated by the ATPG, the coefficients $a_{i j k}$ 's in Eq. (3) is solved so as to generate the sequence of test patterns. The number of stages, $M$, in the 2-D LFSR reflects the hardware complexity. Given a small number of $M$, Eq. (3) is sometimes not solvable; therefore, a modified algorithm is needed. For example, partitioning the original sequence of test patterns into consecutive subsequences of test patterns and then solving Eq. (3) for each subsequence becomes a feasible solution. As the length of each subsequence decreases, Eq. (3) can be solved with an acceptable small number of stages. While the number of LFSR stages decreases by the partitioning sequence, the complexity of the configuration network increases. Hence, it is necessary to optimize the configuration network for a less cost. 


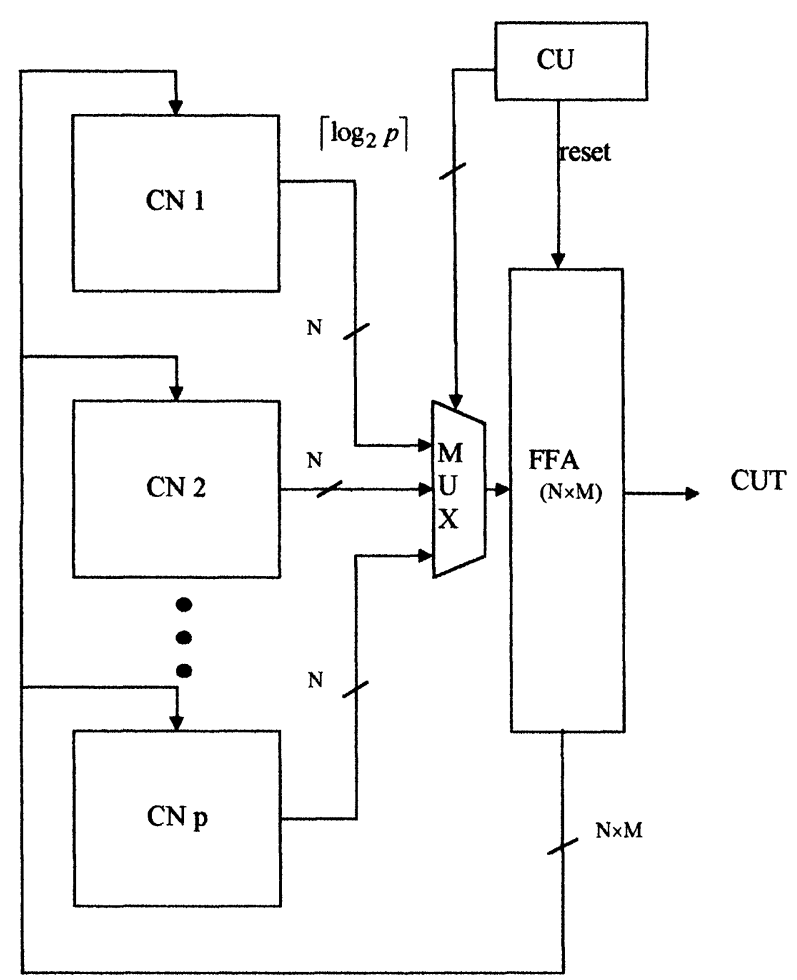

FIGURE 3 Configurable 2-D LFSR based BIST scheme.

The following is an example to show the effectiveness of the configurable 2-D LFSR based generator. Given a deterministic ordered sequence with 18 6-bit patterns with the initial seed $\langle 101010\rangle$, number will be 5 without the configuration structure as shown in Figure 6. The initial patterns of both generators are shown in Figures 5 and 6 . The first test pattern $\langle 101001\rangle$ in this example is generated with the initial pattern $\langle 101010\rangle$ feeding

$\begin{array}{lllllllllllllllllll} & t_{1} & t_{2} & t_{3} & t_{4} & t_{5} & t_{6} & t_{7} & t_{8} & t_{9} & t_{10} & t_{11} & t_{12} & t_{13} & t_{14} & t_{15} & t_{16} & t_{17} & t_{18} \\ 1: & 1 & 0 & 1 & 0 & 0 & 0 & 0 & 1 & 1 & 1 & 0 & 0 & 1 & 0 & 1 & 1 & 1 & 0 \\ 2: & 0 & 1 & 1 & 0 & 1 & 0 & 1 & 1 & 1 & 0 & 0 & 0 & 1 & 0 & 0 & 0 & 0 & 1 \\ 3: & 1 & 1 & 1 & 1 & 0 & 0 & 0 & 1 & 0 & 0 & 1 & 1 & 0 & 0 & 0 & 1 & 0 & 0 \\ 4: & 0 & 1 & 1 & 1 & 1 & 0 & 0 & 0 & 1 & 0 & 0 & 1 & 1 & 0 & 0 & 0 & 1 & 0 \\ 5: & 0 & 0 & 1 & 1 & 1 & 0 & 1 & 1 & 0 & 1 & 0 & 1 & 1 & 1 & 0 & 0 & 1 & 0 \\ 6: & 1 & 0 & 1 & 1 & 1 & 1 & 0 & 0 & 0 & 1 & 1 & 1 & 1 & 1 & 0 & 1 & 0 & 1\end{array}$

both a configurable generator and a non-configurable generator are designed. Figure 5 shows the schematics of the generator with 1-stage registers and two configuration networks, whereas the stage through the configuration network $\mathrm{CN} 1$, and then is shifted into the 2-D LFSR. Other patterns are generated in sequence by following the same procedures until the ninth pattern $\langle 110100\rangle$ is 


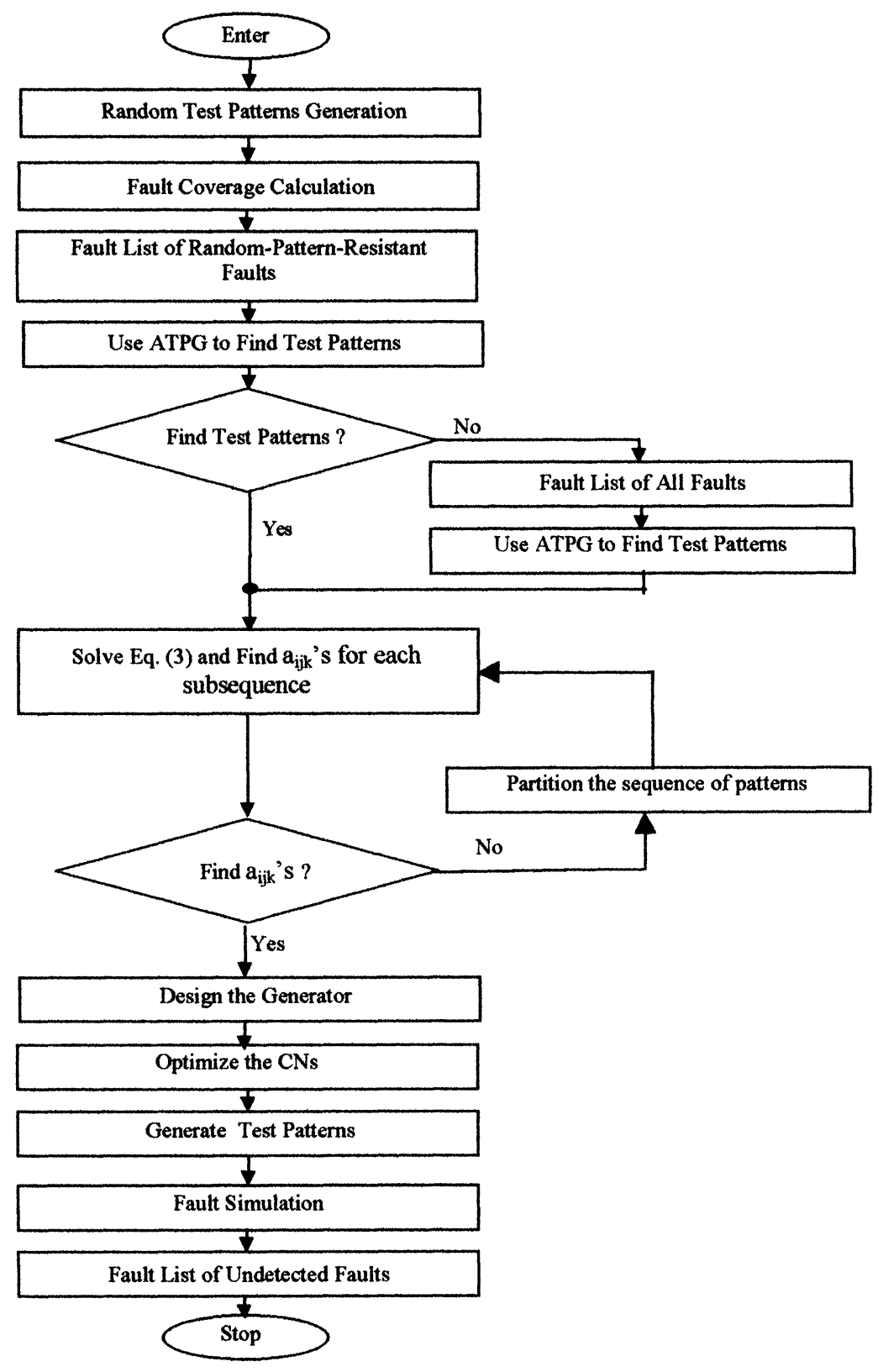

FIGURE 4 Synthesis procedure of a configurable 2-D LFSR.

generated. In this configurable generator, the $\mathrm{CN} 1$ generates the first 9 patterns, $\langle 101001\rangle,\langle 011100\rangle$, $\langle 111111\rangle,\langle 001111\rangle,\langle 010111\rangle,\langle 000001\rangle,\langle 010010\rangle$, $\langle 111010\rangle$ and $\langle 110100\rangle$. The CN2 generates the other 9 patterns, $\langle 100011\rangle,\langle 001001\rangle,\langle 001111\rangle$, $\langle 110111\rangle,\langle 000011\rangle,\langle 100000\rangle,\langle 101001\rangle,\langle 100110\rangle$ and $\langle 010001\rangle$. The tenth pattern is generated with the ninth pattern $\langle 110100\rangle$ feeding through the CN2. The CU with a modulo-9 counter controls this switch. With the optimization on the feedback network of Figure 5, an optimized configurable 2-D LFSR is shown in Figure 7. 


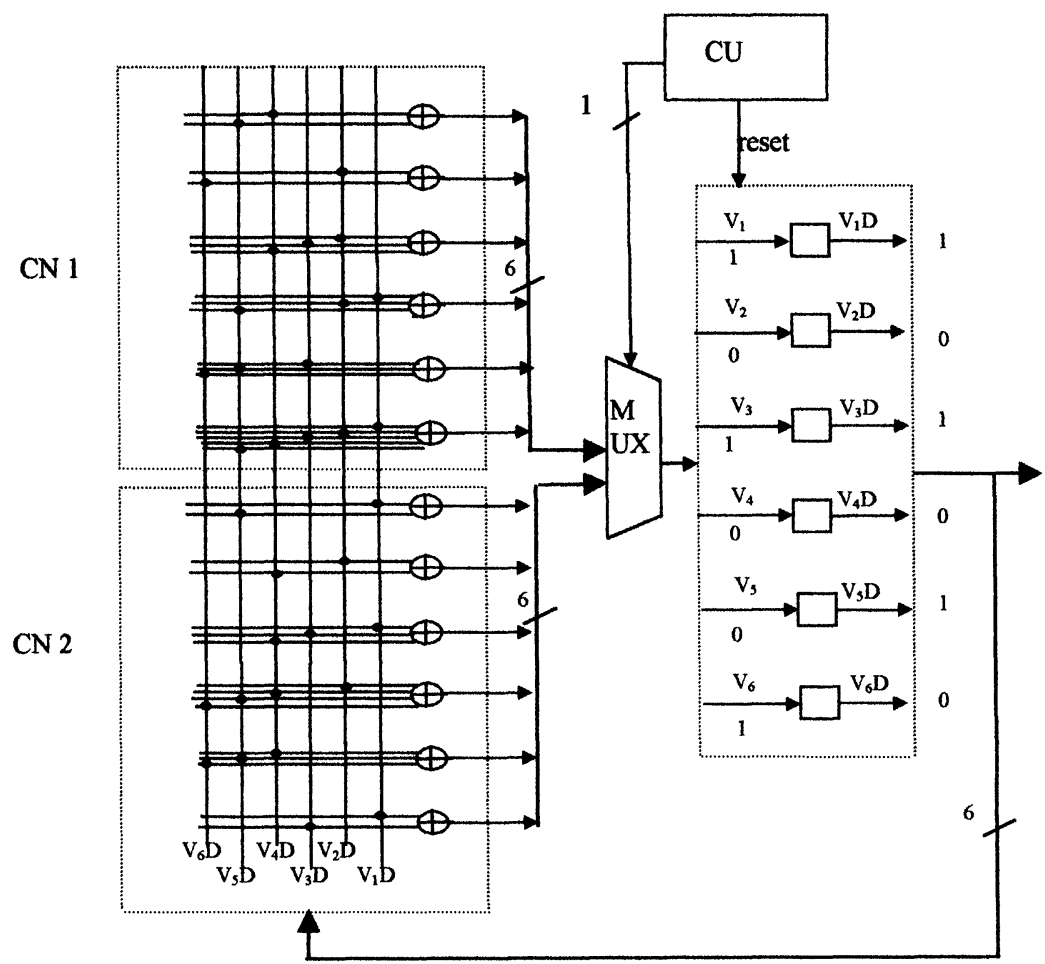

FIGURE 5 A configurable 2-D LFSR based test pattern generator.

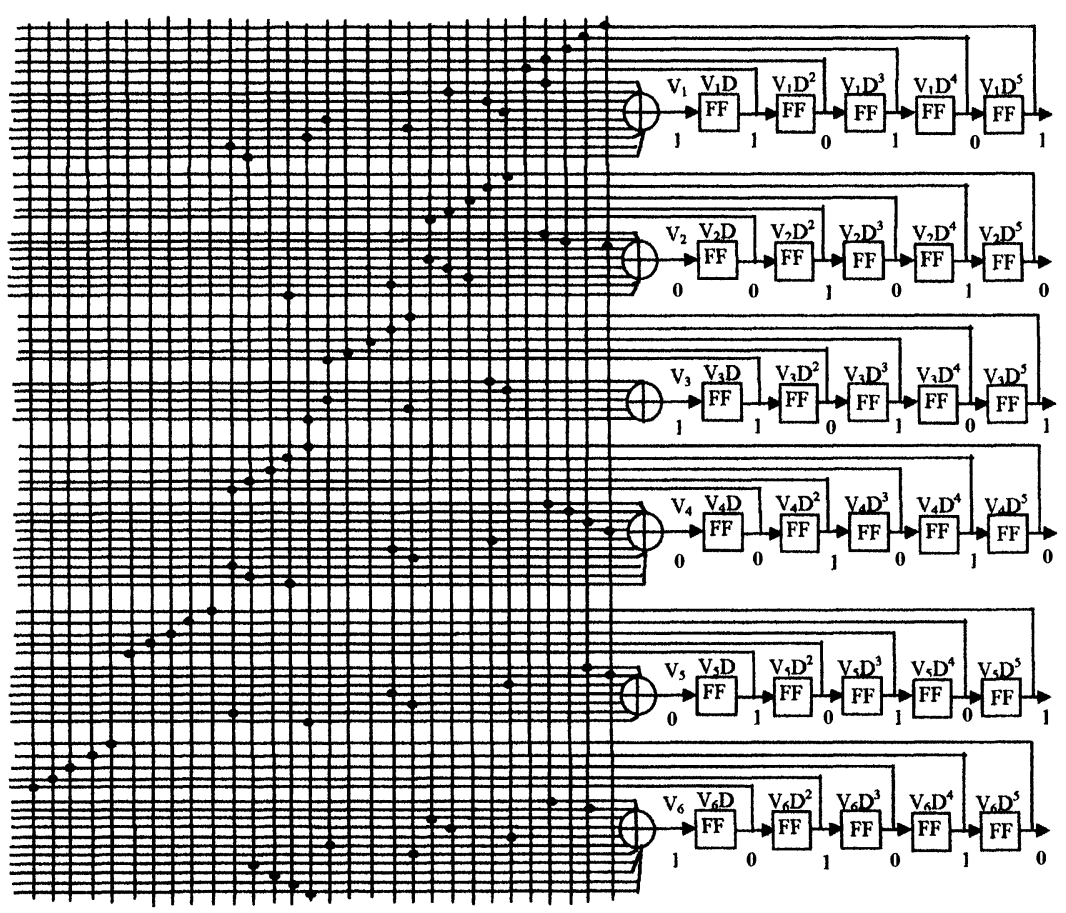

FIGURE 6 A 2-D LFSR test pattern generator. 


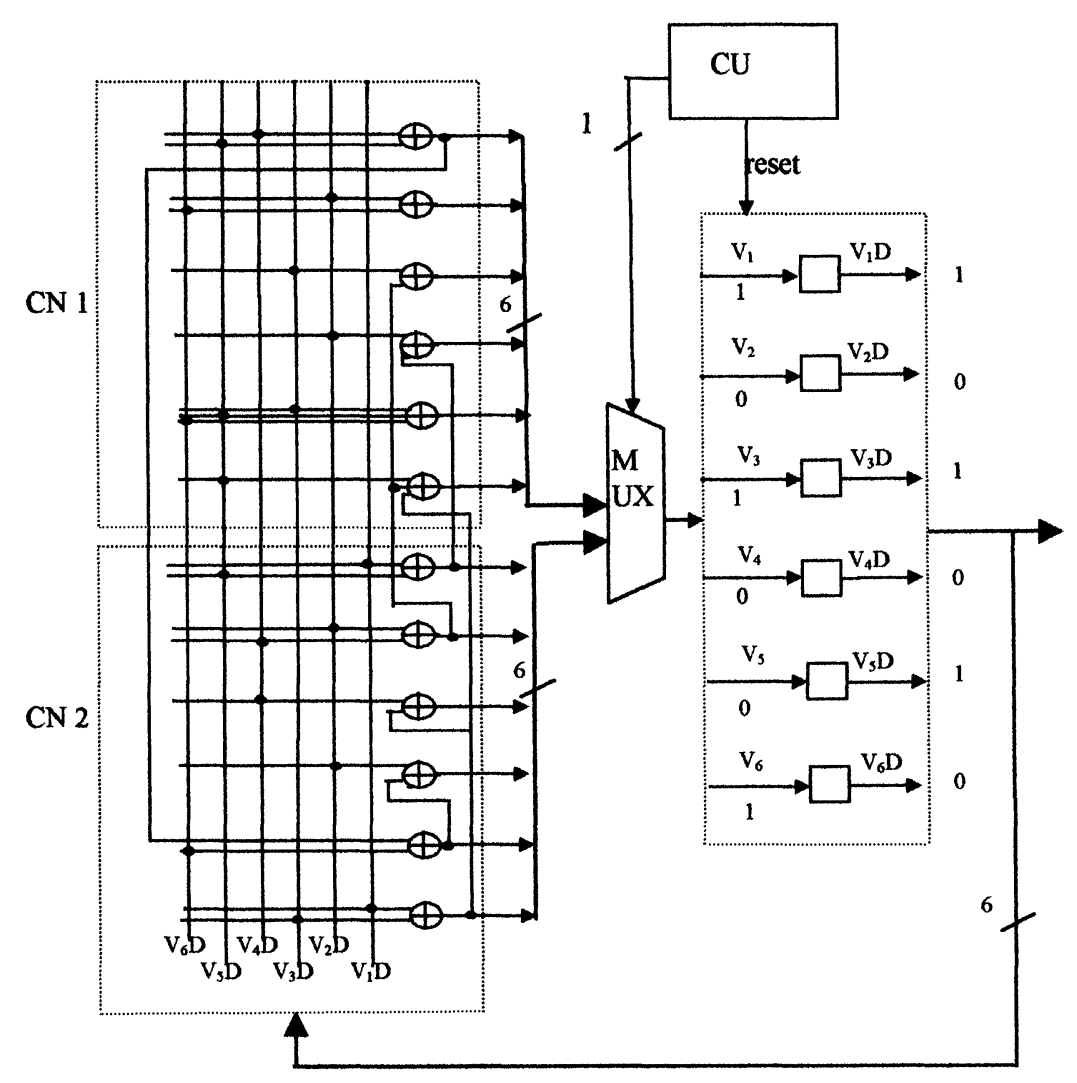

FIGURE 7 An optimized configurable 2-D LFSR based test pattern generator.

\section{EXPERIMENTAL RESULTS}

Five synthesized circuits are used as benchmark circuits to evaluate the configuration structure in the 2-D LFSR scheme. The characteristics of the five circuits are summarized in Table I. Am2910 is a 12-bit microprogram sequencer, which has a stack of depth 5, a stack pointer, a microprogram counter, and a register counter (down counter). A 4-to-1 multiplexer selects the next microprogram

TABLE I Characteristics of five synthesized circuits

\begin{tabular}{lcccc}
\hline Circuits & Seq. depth & FFs & PIs & POs \\
\hline am2910 & 4 & 87 & 20 & 16 \\
mult 16 & 9 & 55 & 18 & 33 \\
div16 & 19 & 50 & 33 & 34 \\
pcont 2 & 3 & 24 & 9 & 8 \\
piir 8 & 5 & 56 & 9 & 8 \\
\hline
\end{tabular}

address from either the input data bus, the stack, the register counter, or the microprogram counter. Mult 16 is a 16-bit 2's complement multiplier, which uses a shift-and-add algorithm. The control unit has been implemented using 3-bit and 4-bit counters. There is one start instruction. Div16 is a 16-bit divider that uses repeated subtraction to perform division. A zero-detect unit checks for the divide-overflow condition and flags the termination of the repeated subtraction loop. There is one control instruction: Start. Pcont 2 is an 8 -bit parallel controller used in DSP applications. This circuit is composed of 8 adders, 2 fixed-point multipliers, and 3 MUXs - all combinational logic. Three registers serve as delay elements. A global reset line resets all the registers, and new data is input at every clock cycle. There is no control unit. Piir8 is an 8-point infinite impulse 
response filter for DSP applications. It has a very regular structure with 7 adders, 8 fixed-point multipliers, 7 8-bit registers, and 7 2-to-1 MUXs. The data input and output buses are 8 bits wide. There is no control unit. A global reset line is the only control signal. In Table II, the configuration and the original non-configuration results of the five benchmark circuits are compared. The first column is the number of embedded deterministic patterns detecting the random-resistant faults for each circuit. The second and third columns give the stage number of shift registers and the total number of flip-flops for the non-configurable test generator. The number of configurations, the stage number of shift registers and the total number of flip-flops for the configurable 2-D LFSR based generator are given in the fourth, fifth and sixth columns. For the am 2910 and mul16 the flip-flops are reduced significantly to $15 \%$ and $14 \%$ respectively. Because the number of embedded patterns for div16, pcont 2 and piir 8 is small, the synthesis procedure results in no reconfiguration. Compared with the other four circuits, div 16 has the largest FFA due to its large number of inputs. The minimum stage number of $M$ can be obtained by increasing the loop times in the branch and bound algorithm while solving Eq. (3) at a cost of CPU time. Table III shows the comparison of the fault. The test patterns generated by HITEC can be either stored in a ROM or scanned in. The results of the conventional LFSR generator are listed in the third column. Column four gives the results from a combination of the conventional LFSR and the additional deterministic ordered patterns. The data in the fifth and last columns are obtained by the 2-D LFSR and the configurable 2-D LFSR based generators respectively. The average number of equivalent faults of five circuits is 9,441 where 3,955 faults are detected by "HITEC" and 6,054 faults are detected by "Random Patterns". Comparing "LFSR", "LFSR + Add", and "2-D LFSR", the experimental results show that the average detected faults by "2-D LFSR" is $5.08 \%$ higher than that of "LFSR + Add" and $8.65 \%$ higher than that of "LFSR". Note that the average number of faults detected by "2-D LFSR" is 5,952, which is only $1.76 \%$ less than that of "Random Patterns". The average number of faults detected by "Configurable 2-D LFSR" is 5,986, which covers about the

TABLE II Hardware comparison of 2-D LFSR and configurable 2-D LFSR

\begin{tabular}{|c|c|c|c|c|c|c|}
\hline \multirow[b]{2}{*}{ Circuits } & \multirow[b]{2}{*}{ Patterns } & \multicolumn{2}{|c|}{ 2-D LFSR } & \multicolumn{3}{|c|}{ Configurable 2-D LFSR } \\
\hline & & Stages & Flip-flops & Confg. & Stages & Flip-flops \\
\hline $\operatorname{am} 2910$ & 30 & 13 & 260 & 2 & 2 & 40 \\
\hline mul16 & 72 & 14 & 252 & 4 & 2 & 36 \\
\hline $\operatorname{div} 16$ & 21 & 10 & 330 & 1 & 2 & 66 \\
\hline pcont 2 & 7 & 2 & 18 & 1 & 2 & 18 \\
\hline piir 8 & 19 & 3 & 27 & 1 & 3 & 27 \\
\hline Total & 149 & - & 887 & - & - & 187 \\
\hline
\end{tabular}

TABLE III Test generation and fault simulation for five synthesized circuits

\begin{tabular}{|c|c|c|c|c|c|c|c|c|c|c|c|c|c|}
\hline \multirow[b]{2}{*}{ Circuits } & \multirow{2}{*}{$\begin{array}{l}\text { Total } \\
\text { faults }\end{array}$} & \multicolumn{2}{|c|}{ HITEC } & \multicolumn{2}{|c|}{$\begin{array}{l}\text { Random } \\
\text { patterns }\end{array}$} & \multicolumn{2}{|c|}{ LFSR } & \multicolumn{2}{|c|}{ LFSR + Add } & \multicolumn{2}{|c|}{ 2-D LFSR } & \multicolumn{2}{|c|}{$\begin{array}{c}\text { Configurable } \\
\text { 2-D LFSR }\end{array}$} \\
\hline & & Det & Vec & Det & Vec & Det & Vec & Det & Vec & Det & $\mathrm{Vec}$ & Det & Vec \\
\hline $\operatorname{am} 2910$ & 2391 & 2142 & 726 & 2155 & $2^{17}$ & 2126 & $2^{17}$ & 2166 & $2^{17}+30$ & 2188 & $2^{17}$ & 2179 & $2^{17}$ \\
\hline mult 16 & 1708 & 1568 & 89 & 1475 & $2^{17}$ & 665 & $2^{17}$ & 1395 & $2^{17}+89$ & 1605 & $2^{17}$ & 1605 & $2^{17}$ \\
\hline $\operatorname{div} 16$ & 2147 & 1676 & 190 & 1718 & $2^{17}$ & 1679 & $2^{17}$ & 1702 & $2^{17}+21$ & 1705 & $2^{17}$ & 1719 & $2^{17}$ \\
\hline pcont 2 & 11272 & 3147 & 7 & 6815 & $2^{17}$ & 6727 & $2^{17}$ & 6727 & $2^{17}+7$ & 6336 & $2^{17}$ & 6518 & $2^{17}$ \\
\hline piir8 & 29689 & 11234 & 19 & 18106 & $2^{17}$ & 16195 & $2^{17}$ & 16331 & $2^{17}+19$ & 17925 & $2^{17}$ & 17913 & $2^{17}$ \\
\hline Avg. & 9441 & 3955 & 209 & 6054 & $2^{17}$ & 5478 & $2^{17}$ & 5664 & $\sim 2^{17}$ & 5952 & $2^{17}$ & 5986 & $2^{17}$ \\
\hline
\end{tabular}


same number of detected faults as the 2-D LFSR generator, but the overall overhead of the FFA has been reduced by $79 \%$.

\section{CONCLUSION}

A BIST scheme based on a configurable 2-D LFSR and a procedure to design a test pattern generator have been presented. Without storage of test patterns and seeds, the 2-D LFSR test pattern generator can generate a sequence of deterministic test patterns as well as pseudo-random patterns. The hardware overhead of this BIST scheme decreased considerably through configuration. The experiment result shows that compared with the non-configurable 2-D LFSR, the number of flip-flops is reduced by $79 \%$ for five synthesized circuits. The average number of faults detected by the configurable 2-D LFSR is $9.27 \%$ higher than that of the conventional LFSR and $0.57 \%$ higher than that of the non-configurable 2-D LFSR.

In order to improve the fault coverage of the configurable 2-D test pattern generator, a set of embedded deterministic patterns with high fault coverage is needed. Logic optimization tools are also necessary to further reduce the hardware overhead of the feedback networks.

\section{References}

[1] Bardell, P., McAnney, W. H. and Savir, J. (1987). Built-in Test for VLSI: Pseudorandom Techniques, Wiley \& Sons, New York.

[2] Chen, C.-I. H. and Yuen, J. (1994). "Automated Synthesis of Pseudo-Exhaustive Test Generator in VLSI BIST Design”, IEEE Trans. on VLSI Systems, 2(3), 273-291, September.

[3] Brglez, F. et al. (1989). "Hardware-Based Weighted Random Pattern Generation for Boundary-Scan", Proc. IEEE Int. Test Conf., pp. 264-274.

[4] Niermann, T. M. and Patel, J. H. (1991). "HITEC: A Test Generation Package for Sequential Circuits," Proc. European Conf. on Design Automation, pp. 213-218.

[5] Hellebrand, S., Tarnick, S., Rajski, J. and Courtois, B. (1992). "Generation of Vector Patterns Through Reseeding of Multiple-Polynomial Linear Feedback Shift Registers", Proc. IEEE Int. Test Conf., pp. 120-129.

[6] Venkataraman, S., Rajski, J., Hellebrand, S. and Tarnick, S. (1993). "An Efficient BIST Scheme Based on Reseeding of Multiple Polynomial Linear Feedback Shift Registers", Proc. Int. Conf. on Computer-Aided Design, pp. 572-577.

[7] Hellebrand, S. et al. (1995). "Built-In Test for Circuits with Scan Based on Reseeding of Multiple-Polynomial Linear Feed-back Shift Registers", IEEE Trans. on Comp., 44(2), 223-233, February.

[8] Yuan, X. and Chen, C.-I. H. (1998). "Automated Synthesis of a Multiple-Sequence Test Generator Using 2-D LFSR", Proc. IEEE International ASIC Conf., pp. $75-79$.

[9] Wagner, K. D., Chin, C. K. and McCluskey, E. J. (1987). "Pseudorandom Testing", IEEE Trans. on Comp., 36(3), $332-343$.

[10] Kagaris, D., Makedon, F. and Tragoudas, S. (1994). "A Method for Pseudo-Exhaustive Test Pattern Generation", IEEE Trans. on CAD, 13(9), 1170-1178.

[11] Wunderlich, H.-J. and Hellebrand, S. (1992). "The Pseudoexhaustive Test for Sequential Circuits", IEEE Trans. on CAD, 11(1), 26-32.

[12] Kapur, R., Patil, S., Snethen, T. J. and Williams, T. W. (1994). "Design of an Efficient Weighted Random Pattern Generation System", Proc. ITC, pp. 491-500.

[13] Muradali, F., Agarwal, V. K. and Nadeau-Dostie, B. (1990). "A New Procedure for Weighted Random Built-in Self-test”, Proc. ITC, pp. 660-669.

\section{Authors' Biographies}

Chien-In Henry Chen received his B.S. degree from the National Taiwan University, Taiwan, in 1981, his M.S. degree from the University of Iowa, Iowa City, in 1986, and his Ph.D. degree from the University of Minnesota, Minneapolis, in 1989, all in electrical engineering. Since joining Wright State University in 1989 he has worked primarily in computer-aided design, timing and testing of very large scale integrated (VLSI) circuits, where he is currently an Associate Professor. He has written over 50 publications in professional journal and conference proceedings and is a technical reviewer for various journals and conferences. He was a technical committee member of the 1995 IEEE International ASIC Conference and Exhibit and was a plenary speaker in the 6th VLSI Design/CAD Symposium.

Yingjie Zhou received his B.S. degree in Electrical Engineering from Tsinghua University, Beijing, China. He was an IC design engineer of Beijing Intelligence Electronic Company, Beijing, China, 1995-98 and is currently a Ph.D. student at Wright State University, Dayton, Ohio. His research interests include VLSI circuit design, design for testability, and built-in self-test. 

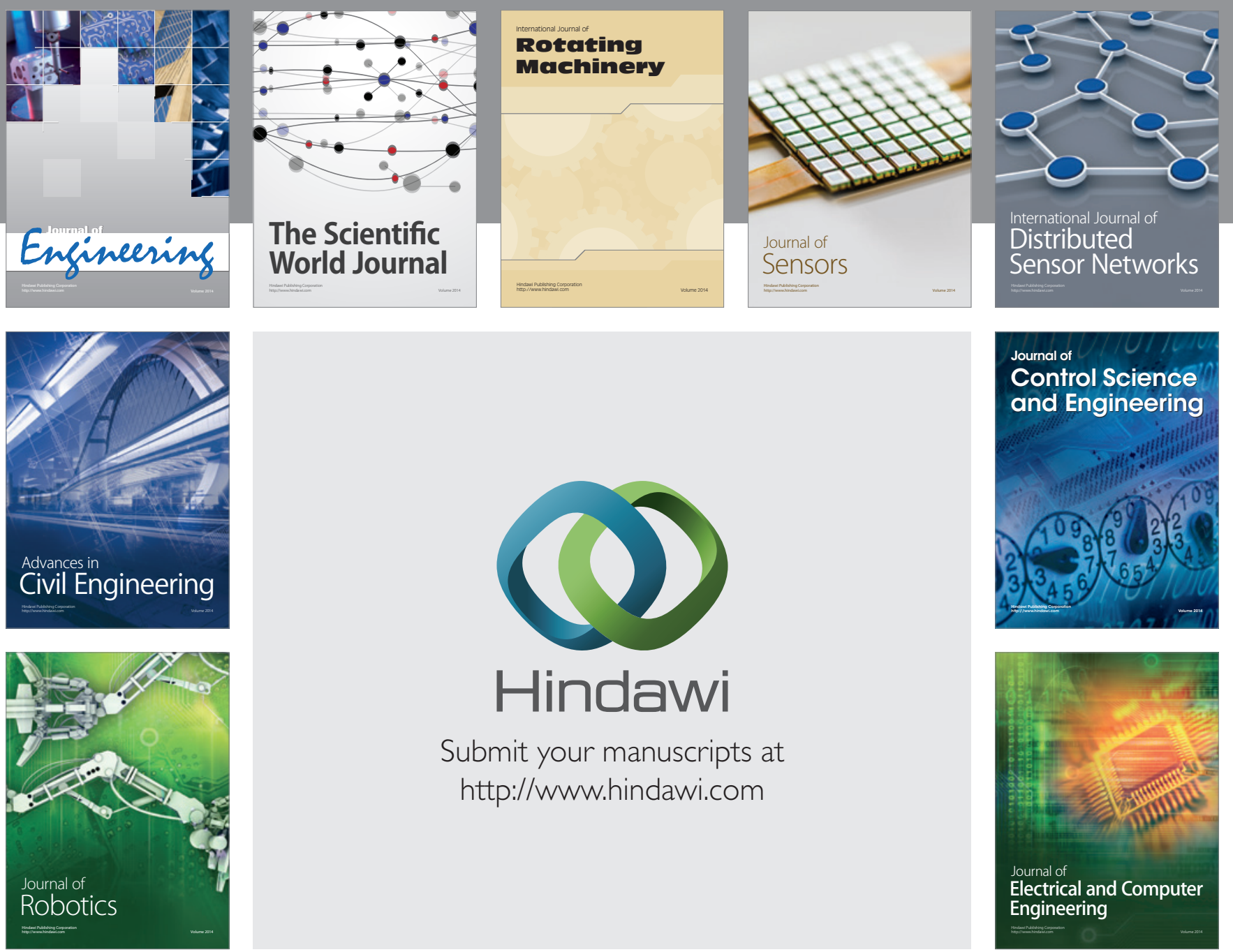

Submit your manuscripts at

http://www.hindawi.com
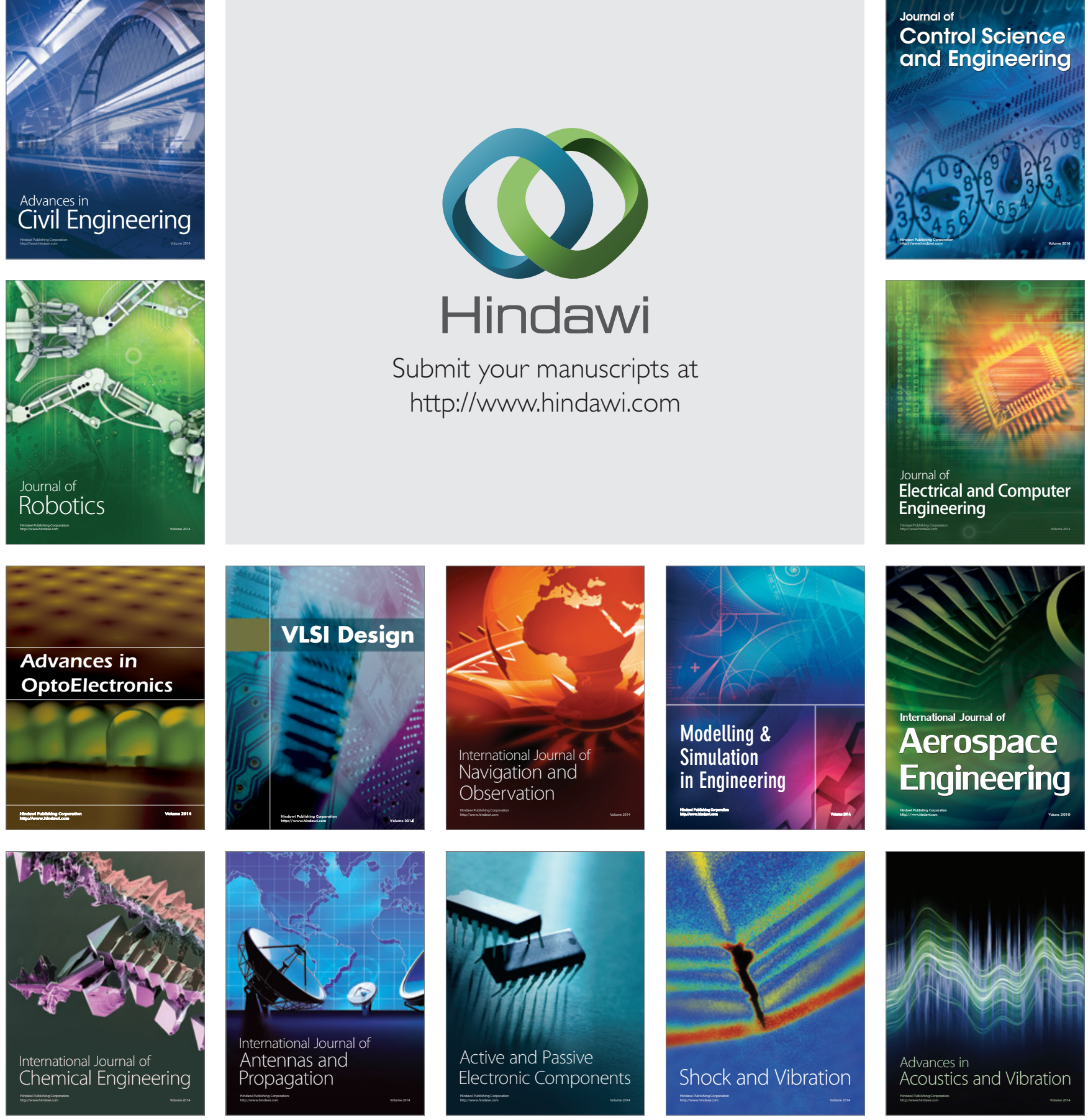